necessary cause for psychosis. This formulation is erroneous and was used, in exactly the same words, many years ago when prospective studies in the UK established the aetiological role of tobacco in lung cancer. In an elementary textbook on statistics, Schwartz (1999) explains that this error arises from the faulty use of the term 'cause', which applies to the domain of certainty, whereas in the domain of uncertainty (i.e. of illness) the definition of a causal factor is that it provokes an increase in risk, as perfectly demonstrated by the authors. One wonders why they make this elementary error. It is unlikely to be due to psychological resistance, as was the case with tobacco smokers at that time. Perhaps they believe that schizophrenia (or psychosis) is a known disease entity, as defined according to international systems of classification (DSM-IV, ICD10) which, unfortunately, continue to exclude substance use from their diagnostic criteria.

Arseneault, L., Cannon, M., Witton, J., et al (2004) Causal association between cannabis and psychosis: examination of the evidence. British Journal of Psychiatry, 184, $110-117$

Schwartz, D. (1999) Le Jeu de la Science et du Hazard. La Statistique et le Vivant. Paris: Flammarion.

J.-P. Luaute 25 Rue de la République, 26100

Romans, France

\section{Child sexual abuse and substance use disorders: role of psychiatric comorbidity}

We read with interest the paper by Spataro et al (2004) considering associations between child sexual abuse and subsequent psychopathology using a prospective cohort design. This study clearly indicates a positive association between child sexual abuse and a range of mental disorders, although not substance use disorders. We think that the authors make an important point in their discussion that this latter absence of an association might be at leastpartly due to their methodology for assessing psychiatric outcome. They implemented a diagnostic hierarchy in such a way that when substance use problems were accompanied by other psychiatric disorders, these comorbid conditions were counted and not the substance use.

It is important for the reader to know that substantial comorbidity between substance use disorders and other psychiatric disorders is consistently reported (e.g. Kessler et al, 1997a). Thus, one could suggest that this prospective study does not demonstrate an association between child sexual abuse and more pure forms of substance use disorders. This would be in line with other findings suggesting a lack of association between childhood trauma (including child sexual abuse) and pure substance use disorders, but a strong relationship between childhood trauma and psychiatric comorbidity in substance use disorders (Kessler et al, 1997b; de Graaf et al, 2002).

\section{De Graaf, R., Bijl, R.V., Smit, F., et al (2002) Risk}

factors for 12-month comorbidity of mood, anxiety, and substance use disorders: findings from The Netherlands Mental Health Survey and Incidence Study. American Journal of Psychiatry, 159, 620-629.

Kessler, R. C., Crum, R. M., Warner, L. A., et al (1997a) Lifetime co-occurrence of DSM-III-R alcohol abuse and dependence with other psychiatric disorders in the national comorbidity survey. Archives of General Psychiatry, 54, 313-321.

Kessler, R. C., Davis, C. G. \& Kendler, K. S. (1997b)

Childhood adversity and adult psychiatric disorder in the US National Comorbidity Survey. Psychological Medicine, 27, $1101-1119$

Spataro, J., Mullen, P. E., Burgess, P. M., et al (2004) Impact of child sexual abuse on mental health Prospective study in males and females. British Journal of Psychiatry, 184, 416-42I.

W. Langeland, $\mathbf{W}$. van den Brink Amsterdam Institute for Addiction Research, Academic Medical Center, University of Amsterdam, Tafelbergweg 25, II05 BC Amsterdam, The Netherlands.

E-mail: w.vandenbrink@amc.uva.nl

\section{Insulin-like growth factors, insulin resistance and schizophrenia}

Abel (Abel, 2004) speculates that imprinting of the gene for insulin-like growth factor-II (IGF-II) as well as other genes may be one pathway through which environmental exposures influence the risk of schizophrenia. We too have hypothesised that factors influencing the growth-hormone-IGF axis may contribute to the well-recognised associations of pre-adult exposures with schizophrenia (Gunnell \& Holly, 2004).

We feel that evidence for a direct role of IGF-I is more compelling than that for IGFII (whose biological functions are poorly understood). Possible pathways for an association with IGF-I lie not only in its role in neurodevelopment but also through its role in neuroprotection following brain damage (e.g. following birth asphyxia, head injury or meningitis) (Gluckman et al, 1998). Insulin-like growth factors exert powerful anti-apoptotic actions and low levels may reduce the survival probability of damaged cells. The influence of IGF-I may extend beyond foetal life as low IGF-I is associated with low birth weight, reduced childhood growth and low body mass index, which are, in turn, associated with the development of psychosis (Wahlbeck et al, 2001; Gunnell et al, 2003). It is therefore possible that low IGF-I levels not only impair neurodevelopment but also render individuals more susceptible to neurodevelopmental insults such as traumatic brain injury and hypoxic brain damage (Gunnell \& Holly, 2004).

Several lines of direct and indirect evidence support a possible role of IGF-I in the aetiology of schizophrenia (Gunnell \& Holly, 2004). Intriguing indirect evidence for the role of IGF-I, as Abel points out, comes from the observation that low levels protect against a range of different cancers (Renehan et al, 2004) and individuals with psychosis, and their families, appear to be at reduced risk of some malignancies. This may well reflect shared genetic influences on IGF levels influencing susceptibility to both schizophrenia and cancer. Evidence for aetiological associations of IGF-II with cancer risk are less consistent than those for IGF-I. A further indirect line of evidence comes from current concern that insulin resistance may both be more common in people with schizophrenia and be precipitated by antipsychotic medication. Prospective studies indicate that low IGF-I levels are associated with the development of insulin resistance (Sandhu et al, 2002). We speculate that the co-occurrence of insulin resistance and psychosis may in part arise through the shared susceptibility of both these disorders associated with low IGF-I levels.

Evaluation of the possible role of the IGF-system in schizophrenia might not only further our understanding of the aetiology of this disorder but also give insights into its prevention and the reduction of comorbidities such as insulin resistance.

Abel, K. M. (2004) Foetal origins of schizophrenia: testable hypotheses of genetic and environmental influences. British Journal of Psychiatry, 184, 383-385.

Gluckman, P. D., Guan, J., Williams, C., et al (1998) Asphyxial brain injury - the role of the IGF system. Molecular and Cellular Endocrinology, 140, 95-99.

Gunnell, D. \& Holly, J. M. P. (2004) Do insulin-like growth factors underlie associations of birth complications, fetal and pre-adult growth with schizophrenia? Schizophrenia Research, 67, 309-311. 